DEPARTMENT OF ENVIRONMENT,

TECHNOLOGY AND TECHNOLOGY MANAGEMENT

\title{
Three-level Equivalent-Estimation Split-Plot Designs Based on Subset and Supplementary Difference Set Designs
}

Kalliopi Mylona, Harrison Macharia \& Peter Goos

\author{
UNI VERSI TY OF ANTWERP \\ Faculty of Applied Economics \\ Stadscampus \\ Prinsstraat 13, B.226 \\ BE-2000 Antwerpen \\ Tel. +32 (0)32654032 \\ Fax +32 (0)32654799 \\ http://www.ua.ac.be/tew
}




\title{
FACULTY OF APPLIED ECONOMI CS
}

\author{
DEPARTMENT OF ENVIRONMENT, \\ TECHNOLOGY AND TECHNOLOGY MANAGEMENT \\ Three-level Equivalent-Estimation Split-Plot \\ Designs Based on Subset and Supplementary \\ Difference Set Designs
}

Kalliopi Mylona, Harrison Macharia \& Peter Goos

RESEARCH PAPER 2011-010

JULY 2011
University of Antwerp, City Campus, Prinsstraat 13, B-2000 Antwerp, Belgium Research Administration - room B.226 phone: (32) 32654032
fax: (32) 32654799
e-mail: joeri.nys@ua.ac.be

The papers can be also found at our website:

www.ua.ac.be/ tew (research > working papers) \&

www.repec.org/ (Research papers in economics - REPEC)

D/ 2011/ 1169/010 


\title{
Three-level Equivalent-Estimation Split-Plot Designs Based on Subset and Supplementary Difference Set Designs
}

\author{
Kalliopi Mylona ${ }^{1}$, Harrison Macharia ${ }^{1}$ and Peter Goos ${ }^{1,2}$ \\ ${ }^{1}$ Faculty of Applied Economics \& StatUa Center for Statistics, Universiteit Antwerpen, \\ Prinsstraat 13, 2000 Antwerpen, Belgium. \\ ${ }^{2}$ Erasmus School of Economics, Erasmus Universiteit Rotterdam, \\ Postbus 1738, 3000 DR Rotterdam, Netherlands.
}

\begin{abstract}
In many industrial experiments, complete randomization of the runs is impossible as, often, they involve factors whose levels are hard or costly to change. In such cases, the split-plot design is a cost-efficient alternative that reduces the number of independent settings of the hard-to-change factors. In general, the use of generalized least squares is required for model estimation based on data from split-plot designs. However, the ordinary least squares estimator is equivalent to the generalized least squares estimator for some split-plot designs, including some second-order split-plot response surface designs. These designs are called equivalent-estimation designs. An important consequence of the equivalence is that basic experimental design software can be used for model estimation. We introduce two new families of equivalent-estimation split-plot designs, one based on subset designs and another based on supplementary difference set designs. The resulting designs complement existing catalogs of equivalent-estimation designs and allow for a more flexible choice of the number of hard-to-change factors, the number of easy-to-change factors, the number and size of whole plots and the total sample size. We show that the newly proposed designs possess good predictive properties when compared to D-optimal split-plot designs.
\end{abstract}

Key Words: generalized least squares, OLS-GLS equivalence, ordinary least squares, response surface design, split-plot design, subset design, supplementary difference set. 


\section{Introduction}

The increased application of design of experiments in industry has resulted in the widespread use of response surface methodology, in general, and response surface designs, in particular, whenever the relationship between a response and settings of a group of quantitative experimental factors is of interest. Response surface designs have been applied for the purpose of developing, improving and optimizing processes in, for instance, industrial and chemical engineering, and biotechnology. A thorough exposition on response surface methodology and designs is given by Khuri and Cornell (1996) and Myers and Montgomery (2002).

In response surface experiments, one is often faced with factors whose levels are hard or expensive to change. This necessitates the use of multi-stratum designs, which provide a more economic and efficient alternative to fully randomized designs. One of the special cases of the multi-stratum designs is the split-plot design. Split-plot designs were first used in agriculture, but they are now widely applied in industry as well. In agricultural experimentation, large portions of land were subdivided into relatively smaller portions known as whole plots, to which each of the possible levels of the whole-plot factors was then randomly assigned. Whole plots were further divided into smaller units, known as split-plots or subplots, and subplot factor levels were then applied randomly to these smaller units. In the context of industrial experiments, the factors whose levels are hard or costly to modify are referred to as hard-to-change factors and act like whole-plot factors. The remaining factors, whose levels can be changed with relative ease, are referred to as easy-to-change factors and act as subplot factors. Therefore, the levels of hard-to-change factors vary from whole plot to whole plot, while the levels of easy-to-change factors vary from subplot to subplot.

For data from response surface split-plot experiments, Letsinger, Myers and Lentner (1996) recommended the use of generalized least squares (GLS), in combination with restricted maximum likelihood (REML), for estimating the experimental factors' effects. This is because the randomization structure of split-plot designs leads to correlated responses, and in the presence of correlated responses, the ordinary least squares (OLS) estimator is less efficient than the GLS estimator.

The design of response surface split-plot experiments has received extensive attention in the literature. Overviews of all the work that has been done can be found in Goos (2002) and Jones and Nachtsheim (2009). A substantial part of that literature has focused on situations where the OLS and GLS estimators for the fixed effects are equivalent. This is a desirable property since, as opposed to the GLS estimator, the OLS estimator does not require knowledge/estimation of the variance components to estimate the parameters. This is important for experimenters who do not have access to modern software for analyzing split-plot data using REML-GLS. It has become common to use the term equivalent-estimation split-plot designs for all split-plot designs for which OLS and GLS 
are equivalent.

The OLS-GLS equivalence for response surface split-plot experiments was first discussed by Letsinger et al. (1996) and Goos and Vandebroek (2001, 2003) showed that a whole range of two-level factorial and fractional factorial designs also guarantees that the OLS and GLS estimates are identical. Vining, Kowalski and Montgomery (2005) and Parker, Kowalski and Vining (2006, 2007a,b) introduced a family of equivalent-estimation splitplot designs for second-order response surface models constructed by modifying central composite and Box-Behnken designs. Furthermore, Goos (2006) and Parker et al. (2007a) reported various instances where the D-optimal split-plot design is an equivalentestimation design. Recently, Macharia and Goos (2010) presented a modified coordinateexchange algorithm for identifying D-efficient equivalent-estimation split-plot designs for second-order response surface models.

In this paper, we present two new families of three-level equivalent-estimation designs. The first is based on subset designs. Subset designs are three-level designs introduced by Gilmour (2006) and further explored by Ahmad and Gilmour (2010). They are obtained by using two-level factorial designs in subsets of factors while the other factors are held at the middle level. Special cases of subset designs are face-centered central composite designs and Box-Behnken designs. The designs we use for the second new family of equivalent-estimation designs are supplementary difference set designs, which are due to Koukouvinos, Mylona and Skountzou (http://www.math.ntua.gr/nckoukouv/). When used in a completely randomized experiment, both subset designs and supplementary difference set designs have properties similar to those of central composite designs and Box-Behnken designs. For instance, all main effects and two-factor interaction effects can be estimated independently with these designs, so that the designs are useful for model selection. Another attractive feature is that the designs are factor-wise balanced, which means that all main-effect estimates have the same variance, all two-factor-interactioneffect estimates have the same variance, and all estimates of quadratic effects have the same variance. The designs are easy to construct, allow the second-order model to be fitted efficiently, and possess attractive projection properties. Moreover, many subset designs and all supplementary difference set designs possess good predictive properties, i.e. they perform very well in terms of the I-optimality criterion. The construction of subset designs is described in detail in Gilmour (2006), while that of supplementary difference set designs has not been published yet. For this reason, we sketch the construction of supplementary difference set designs in the appendix to this paper.

We contrast the two new families of equivalent-estimation split-plot designs with Doptimal split-plot designs obtained using the algorithms of Goos and Vandebroek (2003) and Jones and Goos (2007), and show that it is often possible to construct equivalentestimation designs that outperform the D-optimal designs in terms of precision of prediction, as measured by the I-optimality criterion. This is an important result because, as pointed out by Myers and Montgomery (2002), a good predictive performance is considered more important for a second-order design than a precise estimation of the model 
parameters. This is due to the fact that second-order models and designs are used for optimization, and good prediction and good prediction properties are essential for optimization. Hence, the newly proposed designs do not only possess the equivalent-estimation property, but they have another major selling point.

We first present the split-plot model and discuss the OLS-GLS equivalence condition. In Section 3, we show how to construct the new equivalent-estimation designs and provide several examples. In Section 4, we give two lists of equivalent-estimation designs we found. These lists are by no mean exhaustive, but they provide an idea about how broad the range of equivalent-estimation designs is that can be constructed from the subset and supplementary difference set designs. In the lists, we report the D- and I-efficiencies of the designs to demonstrate the practical usefulness of the designs. We end the paper with a summary of the results.

\section{The Model}

In this section, we present the model for data from split-plot response surface designs. In general, the response $Y_{i j}$ of the $j$ th run in the $i$ th whole plot can be written as

$$
\begin{aligned}
y_{i j}= & \beta_{0}+\sum_{k=1}^{M_{w}}\left(\beta_{k}^{w} w_{k i}+\beta_{k k}^{w} w_{k i}^{2}\right)+\sum_{k=1}^{M_{s}}\left(\beta_{k}^{s} s_{k i j}+\beta_{k k}^{s} s_{k i j}^{2}\right)+\sum_{k=1}^{M_{w}} \sum_{l=k+1}^{M_{w}} \beta_{k l}^{w w} w_{k i} w_{l i} \\
& +\sum_{k=1}^{M_{s}} \sum_{l=k+1}^{M_{s}} \beta_{k l}^{s s} s_{k i j} s_{l i j}+\sum_{k=1}^{M_{w}} \sum_{l=1}^{M_{s}} \beta_{k l}^{w s} w_{k i} s_{l i j}+\gamma_{i}+\epsilon_{i j},
\end{aligned}
$$

where $w_{k i}$ is the level of the $k$ th hard-to-change factor in the $i$ th whole plots, $M_{w}$ is the number of hard-to-change factors, $s_{k i j}$ is the level of the $k$ th easy-to-change factor at the $j$ th run in the $i$ th whole plot, $M_{s}$ is the number of easy-to-change factors, $\beta_{0}$ is the intercept, $\beta_{k}^{w}$ is the main effect of the $k$ th hard-to-change factor, $\beta_{k k}^{w}$ is the quadratic effect of the $k$ th hard-to-change factor, $\beta_{k}^{s}$ is the main effect of the $k$ th easy-to-change factor, $\beta_{k k}^{s}$ is the quadratic effect of the $k$ th easy-to-change factor, $\beta_{k l}^{w w}$ is the interaction effect involving the $k$ th and the $l$ th hard-to-change factor, $\beta_{k l}^{s s}$ is the interaction effect involving the $k$ th and the $l$ th easy-to-change factor, $\beta_{k l}^{w s}$ is the interaction effect involving the $k$ th hard-to-change factor and the $l$ th easy-to-change factor, $\gamma_{i}$ is the random effect of the $i$ the whole plot and $\epsilon_{i j}$ is the random error of the $j$ th response in the $i$ th whole plot. We denote the total number of factors, $M_{w}+M_{s}$, by $M$. The total number of model parameters is $p=1+2 M+M(M-1) / 2$.

For a design with $N$ runs and $b$ whole plots of $n$ runs, the matrix form of the model is given by

$$
\boldsymbol{Y}=\mathbf{X} \boldsymbol{\beta}+\mathrm{Z} \gamma+\boldsymbol{\epsilon}
$$


where $\boldsymbol{Y}$ is the $N \times 1$ response vector, $\mathbf{X}$ is the $N \times p$ model matrix, $\boldsymbol{\beta}$ is a $p$-dimensional vector containing the intercept and all the factors' main effects, quadratic effects and interaction effects, $\mathbf{Z}$ is an $N \times b$ matrix of zeros and ones whose $(i, j)$ th element is one if the $i$ th observation was obtained in whole plot $j$ and zero otherwise, $\gamma$ is the $b$ dimensional vector containing the whole-plot random effects, and $\boldsymbol{\epsilon}$ is the $N$-dimensional vector of the random errors. The random errors $\boldsymbol{\epsilon}$ and $\gamma$ are assumed to be independent with zero means and variance-covariance matrix $\sigma_{\epsilon}^{2} \mathbf{I}_{N}$ and $\sigma_{\gamma}^{2} \mathbf{I}_{b}$, respectively, where $\mathbf{I}_{N}$ and $\mathbf{I}_{b}$ are identity matrices of sizes $N$ and $b$. Therefore, the variance-covariance matrix of the split-plot model is given by

$$
\mathbf{V}=\sigma_{\epsilon}^{2} \mathbf{I}_{N}+\sigma_{\gamma}^{2} \mathbf{Z} \mathbf{Z}^{\prime}
$$

In general, the total number of runs $N$, the number of whole plots $b$ and the number of runs within a whole plot, $n$, is dictated by budget constraints and logistical considerations such as batch sizes and oven sizes.

The OLS estimator of the factor effects in $\boldsymbol{\beta}$ is

$$
\left(\mathbf{X}^{\prime} \mathbf{X}\right)^{-1} \mathbf{X}^{\prime} \mathbf{Y}
$$

but, for data from split-plot experiments, the GLS estimator

$$
\left(\mathbf{X}^{\prime} \mathbf{V}^{-1} \mathbf{X}\right)^{-1} \mathbf{X}^{\prime} \mathbf{V}^{-1} \mathbf{Y}
$$

is generally more efficient.

The problem with using the GLS estimator is that it requires estimating $\sigma_{\gamma}^{2}$ and $\sigma_{\epsilon}^{2}$. The most broadly applicable method to do so is to use REML estimation. Unfortunately, this estimation method has not been implemented yet in some commonly used software packages for industrial statistics. This explains why some researchers have focused on finding equivalent-estimation split-plot designs that circumvent the need for estimating $\sigma_{\gamma}^{2}$ and $\sigma_{\epsilon}^{2}$ to obtain GLS estimates of the factor effects. While this is certainly useful, it is important to realize that estimating $\sigma_{\gamma}^{2}$ and $\sigma_{\epsilon}^{2}$ remains necessary for performing proper significance tests. As a result, equivalent-estimation designs are not a panacea that overcomes all the shortcomings of certain software packages when it comes to analyzing data from split-plot experiments.

McElroy (1967) showed that the necessary and sufficient condition for the equality of OLS and GLS estimators is the existence of a $p \times p$ nonsingular matrix $\mathbf{F}$, such that

$$
\mathrm{XF}=\mathrm{VX} \text {. }
$$

An alternative expression for the OLS-GLS equivalence condition,

$$
\left(\mathbf{X}^{\prime} \mathbf{X}\right)^{-1} \mathbf{X}^{\prime}=\left(\mathbf{X}^{\prime} \mathbf{V}^{-1} \mathbf{X}\right)^{-1} \mathbf{X}^{\prime} \mathbf{V}^{-1}
$$

tailored to split-plot designs, was derived by Parker et al. (2007a) and discussed by Macharia and Goos (2010). It is given as

$$
\mathbf{X}\left(\mathbf{X}^{\prime} \mathbf{X}\right)^{-1} \mathbf{X}^{\prime} \mathbf{Z} \mathbf{Z}^{\prime} \mathbf{X}=\mathbf{Z} \mathbf{Z}^{\prime} \mathbf{X}
$$




\section{Design Construction Strategies}

In this section, we describe how we use two classes of response surface designs to construct split-plot designs for which the OLS and GLS estimators are equivalent. The first class of response surface designs that we use are the three-level subset designs introduced by Gilmour (2006). The designs are called subset designs because they are formed by combining two or more subsets from the $3^{M}$ factorial designs. The second class of designs that we study is constructed from supplementary difference set designs. A key feature of the supplementary difference set designs is that they do not involve the vertices of the unit cube. These designs, as well as the split-plot designs we obtain from them, are therefore suitable for a spherical design region. Some of the split-plot designs we derive from the subset designs are also suitable for a spherical design region, while others are more useful for a cuboidal design region.

\subsection{Equivalent-Estimation Split-Plot Designs Based on Subset Designs}

The $3^{M}$ factorial design consists of $M+1$ different subsets $S_{r}, r=0, \ldots, M$, where each subset contains the runs that have $r$ different factors acting at their high or low level and the $M-r$ remaining factors acting at their middle level. For the clarity of our exposition, we list the $M_{w}$ hard-to-change factors first, for any design discussed in this paper.

The first step we need to take to construct the equivalent-estimation split-plot designs is to sort the runs within every subset $S_{r}$ in increasing order of the hard-to-change factors' levels. This yields groups of runs that form whole plots of unequal sizes, each of which has an orthogonal subplot design. The next step then is to split these whole plots into smaller ones that still have orthogonal subplot designs. In case a design is made using the subset $S_{1}$, the axial runs for the subplot factors should be kept together to maintain the orthogonality of the subplot design. When they are included in the designs, the axial runs for the whole-plot factors need to be replicated to obtain whole plots of equal sizes. For the same reason, all designs involving the center run must have the center run replicated. We now describe two examples to illustrate the construction of equivalent-estimation split-plot designs based on subset designs.

Example 1. A design for one whole-plot factor $w_{1}$ and two subplot factors $s_{1}$ and $s_{2}$. Table 1 shows the four available subsets from the $3^{3}$ factorial design, which we use as the starting point for our construction. It is clear from the table that the subset $S_{3}$ includes the runs where all factors act at either their low or their high level, while the subset $S_{2}$ has all the runs in which two factors take either level -1 or +1 . The set $S_{1}$ contains the axial runs, which have only one factor whose level is nonzero. Finally, $S_{0}$ is 
a singleton containing the center point. In order to obtain split-plot designs, the runs

Table 1: Four subsets for the $3^{3}$ factorial design

\begin{tabular}{rrr|rrr|rrr|rrr}
\hline \multicolumn{3}{c|}{$S_{3}$} & \multicolumn{3}{|c|}{$S_{2}$} & \multicolumn{3}{|c|}{$S_{1}$} & \multicolumn{3}{|c}{$S_{0}$} \\
\hline$w_{1}$ & $s_{1}$ & $s_{2}$ & $w_{1}$ & $s_{1}$ & $s_{2}$ & $w_{1}$ & $s_{1}$ & $s_{2}$ & $w_{1}$ & $s_{1}$ & $s_{2}$ \\
\hline-1 & -1 & -1 & -1 & -1 & 0 & -1 & 0 & 0 & 0 & 0 & 0 \\
-1 & -1 & 1 & -1 & 1 & 0 & 1 & 0 & 0 & & & \\
-1 & 1 & -1 & 1 & -1 & 0 & 0 & -1 & 0 & & & \\
-1 & 1 & 1 & 1 & 1 & 0 & 0 & 1 & 0 & & & \\
1 & -1 & -1 & -1 & 0 & -1 & 0 & 0 & -1 & & & \\
1 & -1 & 1 & -1 & 0 & 1 & 0 & 0 & 1 & & & \\
1 & 1 & -1 & 1 & 0 & -1 & & & & & & \\
1 & 1 & 1 & 1 & 0 & 1 & & & & & & \\
& & & 0 & -1 & -1 & & & & & & \\
& & & 0 & -1 & 1 & & & & & & \\
& & & 0 & 1 & -1 & & & & & & \\
& & & 0 & 1 & 1 & & & & & & \\
\hline
\end{tabular}

within each subset $S_{i}$ are sequentially sorted in ascending order of the levels of $w_{1}, s_{1}$ and $s_{2}$. This results in Table 2, where we gave every run a unique label: the label $S_{i j}$ refers to the $j$ th run in subset $S_{i}$. The sorted runs in subsets $S_{3}$ and $S_{2}$ in this table appear in groups of four.

Using four as a whole-plot size, we obtained the following three equivalent-estimation split-plot designs:

1. a 20-run design with five whole plots of size four, utilizing the subsets $S_{3}$ and $S_{2}$ :

$$
\begin{gathered}
\left\{S_{31}, S_{32}, S_{33}, S_{34}\right\},\left\{S_{35}, S_{36}, S_{37}, S_{38}\right\},\left\{S_{21}, S_{22}, S_{23}, S_{24}\right\}, \\
\left\{S_{25}, S_{26}, S_{27}, S_{28}\right\},\left\{S_{29}, S_{2,10}, S_{2,11}, S_{2,12}\right\}
\end{gathered}
$$

2. a 20-run design with five whole plots of size four, utilizing the subsets $S_{3}$ and $S_{1}$ :

$$
\begin{gathered}
\left\{S_{31}, S_{32}, S_{33}, S_{34}\right\},\left\{S_{35}, S_{36}, S_{37}, S_{38}\right\},\left\{S_{11}, S_{11}, S_{11}, S_{11}\right\} \\
\left\{S_{12}, S_{13}, S_{14}, S_{15}\right\},\left\{S_{16}, S_{16}, S_{16}, S_{16}\right\}
\end{gathered}
$$

3. a 16-run design with four whole plots of size four, utilizing the subsets $S_{2}$ and $S_{0}$ :

$$
\left\{S_{21}, S_{22}, S_{23}, S_{24}\right\},\left\{S_{25}, S_{26}, S_{27}, S_{28}\right\},\left\{S_{29}, S_{2,10}, S_{2,11}, S_{2,12}\right\},\left\{S_{01}, S_{01}, S_{01}, S_{01}\right\}
$$


Table 2: Sorted subsets for the $3^{3}$ factorial design

\begin{tabular}{r|rrr|rrr|rrr|rrr}
\hline$j$ & \multicolumn{3}{|c|}{$S_{3}$} & \multicolumn{3}{c|}{$S_{2}$} & \multicolumn{3}{|c|}{$S_{1}$} & \multicolumn{3}{|c}{$S_{0}$} \\
\hline & $w_{1}$ & $s_{1}$ & $s_{2}$ & $w_{1}$ & $s_{1}$ & $s_{2}$ & $w_{1}$ & $s_{1}$ & $s_{2}$ & $w_{1}$ & $s_{1}$ & $s_{2}$ \\
\hline 1 & -1 & -1 & -1 & -1 & -1 & 0 & -1 & 0 & 0 & 0 & 0 & 0 \\
2 & -1 & -1 & 1 & -1 & 1 & 0 & 0 & -1 & 0 & & & \\
3 & -1 & 1 & -1 & -1 & 0 & -1 & 0 & 1 & 0 & & & \\
4 & -1 & 1 & 1 & -1 & 0 & -1 & 0 & 0 & -1 & & & \\
5 & 1 & -1 & -1 & 0 & -1 & -1 & 0 & 0 & 1 & & & \\
6 & 1 & -1 & 1 & 0 & -1 & 1 & 1 & 0 & 0 & & & \\
7 & 1 & 1 & -1 & 0 & 1 & -1 & & & & & & \\
8 & 1 & 1 & 1 & 0 & 1 & 1 & & & & & & \\
9 & & & & 1 & -1 & 0 & & & & & & \\
10 & & & & 1 & 1 & 0 & & & & & & \\
11 & & & & 1 & 1 & 0 & & & & & & \\
12 & & & & 1 & 0 & 1 & & & & & & \\
\hline
\end{tabular}

The first two split-plot designs include five whole plots, each containing four runs, whereas the third design includes four whole plots each of which is of size four too. The first design involves runs from the subsets $S_{3}$ and $S_{2}$. None of the runs is replicated in the design. The second design involves runs from the subsets $S_{3}$ and $S_{1}$, and each of the axial points for the whole-plot factor $\left(S_{11}\right.$ and $\left.S_{16}\right)$ is replicated four times within one whole plot. The axial points in the design are therefore treated exactly as in the designs proposed by Vining et al. (2005). The third design is constructed using the subsets $S_{2}$ and $S_{0}$. Therefore, the third design has the same design points as a Box-Behnken design. It has four replicates of the center point in its fourth whole plot. Therefore, the center point is used in a similar fashion as in the design described in Vining et al. (2005).

The runs used in the first and second design are different from the runs of either a central composite design or a Box-Behnken design. Therefore, the split-plot designs based on subset designs generalize the set of equivalent-estimation designs proposed by Vining et al. (2005), who consider only central composite designs and Box-Behnken designs. It should be pointed out that these two designs are suitable for a cuboidal design region because they include the vertices of the unit cube, whereas the third design is more suitable for a spherical design region.

This example shows that equivalent-estimation split-plot designs derived from subset de- 
signs offer additional flexibility to the experimenter. As a matter of fact, in some experiments, the budgeted numbers of runs and whole plots are different from those required to run the equivalent-estimation central composite or Box-Behnken designs suggested by Vining et al. (2005) and Parker et al. (2006,2007a,b). So, the new family of equivalentestimation designs offers useful new design options to experimenters who do not want to resort to optimal experimental design approaches and who wish to use ordinary rather than generalized least squares estimation.

Example 2. A design for two whole-plot factors $w_{1}$ and $w_{2}$ and two subplot factors $s_{1}$ and $s_{2}$. Table 3 shows the five available subsets from the $3^{4}$ factorial design, with the runs sorted in ascending order of $w_{1}$ and $w_{2}$. The runs in the sorted subsets $S_{4}$ and $S_{3}$ in the table again appear in groups of size four. Using four as a whole-plot size, we obtained the following four equivalent-estimation split-plot designs:

1. a 36-run design with nine whole plots of four runs, using the subsets $S_{4}$ and $S_{1}$ :

$$
\begin{gathered}
\left\{S_{41}, S_{42}, S_{43}, S_{44}\right\},\left\{S_{45}, S_{46}, S_{47}, S_{48}\right\},\left\{S_{49}, S_{4,10}, S_{4,11}, S_{4,12}\right\},\left\{S_{4,13}, S_{4,14}, S_{4,15}, S_{4,16}\right\} \\
\left\{S_{11}, S_{11}, S_{11}, S_{11}\right\},\left\{S_{12}, S_{12}, S_{12}, S_{12}\right\},\left\{S_{17}, S_{17}, S_{17}, S_{17}\right\},\left\{S_{18}, S_{18}, S_{18}, S_{18}\right\} \\
\left\{S_{13}, S_{14}, S_{15}, S_{16}\right\}
\end{gathered}
$$

2. a 36-run design with nine whole plots of four runs, using the subsets $S_{3}$ and $S_{0}$ :

$$
\begin{gathered}
\left\{S_{31}, S_{32}, S_{33}, S_{34}\right\},\left\{S_{35}, S_{36}, S_{37}, S_{38}\right\},\left\{S_{39}, S_{3,10}, S_{3,11}, S_{3,12}\right\},\left\{S_{3,13}, S_{3,14}, S_{3,15}, S_{3,16}\right\} \\
\left\{S_{3,17}, S_{3,18}, S_{3,19}, S_{3,20}\right\},\left\{S_{3,21}, S_{3,22}, S_{3,23}, S_{3,24}\right\},\left\{S_{3,25}, S_{3,26}, S_{3,27}, S_{3,28}\right\} \\
\left\{S_{3,29}, S_{3,30}, S_{3,31}, S_{3,32}\right\},\left\{S_{01}, S_{01}, S_{01}, S_{01}\right\}
\end{gathered}
$$

3. a 72-run design with eighteen whole plots of four runs, combining the two 36-run designs, and, hence, utilizing the subsets $S_{4}, S_{3}, S_{1}$ and $S_{0}$,

4. a 68-run design with seventeen whole plots of four runs, obtained by dropping the whole plot containing the center points from the previous design.

It is also possible to construct equivalent-estimation designs involving whole plots of eight instead of four runs. For instance, the following 72-run equivalent-estimation split-plot design with nine whole plots of size eight is obtained by combining the four-run whole plots of the two 36-run designs into whole plots of size eight:

$$
\begin{gathered}
\left\{S_{41}, S_{42}, S_{43}, S_{44}, S_{31}, S_{32}, S_{33}, S_{34}\right\},\left\{S_{45}, S_{46}, S_{47}, S_{48}, S_{39}, S_{3,10}, S_{3,11}, S_{3,12}\right\}, \\
\left\{S_{49}, S_{4,10}, S_{4,11}, S_{4,12}, S_{3,21}, S_{3,22}, S_{3,23}, S_{3,24}\right\},\left\{S_{4,13}, S_{4,14}, S_{4,15}, S_{4,16}, S_{3,29}, S_{3,30}, S_{3,31}, S_{3,32}\right\}, \\
\left\{S_{11}, S_{11}, S_{11}, S_{11}, S_{35}, S_{36}, S_{37}, S_{38}\right\},\left\{S_{12}, S_{12}, S_{12}, S_{12}, S_{3,13}, S_{3,14}, S_{3,15}, S_{3,16}\right\}, \\
\left\{S_{17}, S_{17}, S_{17}, S_{17}, S_{3,17}, S_{3,18}, S_{3,19}, S_{3,20}\right\},\left\{S_{18}, S_{18}, S_{18}, S_{18}, S_{3,25}, S_{3,26}, S_{3,27}, S_{3,28}\right\} . \\
\left\{S_{13}, S_{14}, S_{15}, S_{16}, S_{01}, S_{01}, S_{01}, S_{01}\right\} .
\end{gathered}
$$


Table 3: Sorted subsets for the $3^{4}$ factorial design

\begin{tabular}{|c|c|c|c|c|c|c|c|c|c|c|c|c|c|c|c|c|c|c|c|c|}
\hline \multirow[b]{2}{*}{$j$} & \multicolumn{4}{|c|}{$S_{4 j}$} & \multicolumn{4}{|c|}{$S_{3 j}$} & \multicolumn{4}{|c|}{$S_{2 j}$} & \multicolumn{4}{|c|}{$S_{1 j}$} & \multicolumn{4}{|c|}{$S_{0 j}$} \\
\hline & $w_{1}$ & $w_{2}$ & $s_{1}$ & $s_{2}$ & $w_{1}$ & $w_{2}$ & $s_{1}$ & $s_{2}$ & $w_{1}$ & $w_{2}$ & $s_{1}$ & $s_{2}$ & $w_{1}$ & $w_{2}$ & $s_{1}$ & $s_{2}$ & $w_{1}$ & $w_{2}$ & $s_{1}$ & $s_{2}$ \\
\hline 1 & -1 & -1 & -1 & -1 & -1 & -1 & -1 & 0 & -1 & -1 & 0 & 0 & -1 & 0 & 0 & 0 & 0 & 0 & 0 & 0 \\
\hline 2 & -1 & -1 & -1 & 1 & -1 & -1 & 1 & 0 & -1 & 0 & -1 & 0 & 0 & -1 & 0 & 0 & & & & \\
\hline 3 & -1 & -1 & 1 & -1 & -1 & -1 & 0 & -1 & -1 & 0 & 1 & 0 & 0 & 0 & -1 & 0 & & & & \\
\hline 4 & -1 & -1 & 1 & 1 & -1 & -1 & 0 & 1 & -1 & 0 & 0 & -1 & 0 & 0 & 1 & 0 & & & & \\
\hline 5 & -1 & 1 & -1 & -1 & -1 & 0 & -1 & -1 & -1 & 0 & 0 & 1 & 0 & 0 & 0 & -1 & & & & \\
\hline 6 & -1 & 1 & -1 & 1 & -1 & 0 & -1 & 1 & -1 & 1 & 0 & 0 & 0 & 0 & 0 & 1 & & & & \\
\hline 7 & -1 & 1 & 1 & -1 & -1 & 0 & 1 & -1 & 0 & -1 & -1 & 0 & 0 & 1 & 0 & 0 & & & & \\
\hline 8 & -1 & 1 & 1 & 1 & -1 & 0 & 1 & 1 & 0 & -1 & 1 & 0 & 1 & 0 & 0 & 0 & & & & \\
\hline 9 & 1 & -1 & -1 & -1 & -1 & 1 & -1 & 0 & 0 & -1 & 0 & -1 & & & & & & & & \\
\hline 10 & 1 & -1 & -1 & 1 & -1 & 1 & 1 & 0 & 0 & -1 & 0 & 1 & & & & & & & & \\
\hline 11 & 1 & -1 & 1 & -1 & -1 & 1 & 0 & -1 & 0 & 0 & -1 & -1 & & & & & & & & \\
\hline 12 & 1 & -1 & 1 & 1 & -1 & 1 & 0 & 1 & 0 & 0 & -1 & 1 & & & & & & & & \\
\hline 13 & 1 & 1 & -1 & -1 & 0 & -1 & -1 & -1 & 0 & 0 & 1 & -1 & & & & & & & & \\
\hline 14 & 1 & 1 & -1 & 1 & 0 & -1 & -1 & 1 & 0 & 0 & 1 & 1 & & & & & & & & \\
\hline 15 & 1 & 1 & 1 & -1 & 0 & -1 & 1 & -1 & 0 & 1 & -1 & 0 & & & & & & & & \\
\hline 16 & 1 & 1 & 1 & 1 & 0 & -1 & 1 & 1 & 0 & 1 & 1 & 0 & & & & & & & & \\
\hline 17 & & & & & 0 & 1 & -1 & -1 & 0 & 1 & 0 & -1 & & & & & & & & \\
\hline 18 & & & & & 0 & 1 & -1 & 1 & 0 & 1 & 0 & 1 & & & & & & & & \\
\hline 19 & & & & & 0 & 1 & 1 & -1 & 1 & -1 & 0 & 0 & & & & & & & & \\
\hline 20 & & & & & 0 & 1 & 1 & 1 & 1 & 0 & -1 & 0 & & & & & & & & \\
\hline 21 & & & & & 1 & -1 & -1 & 0 & 1 & 0 & 1 & 0 & & & & & & & & \\
\hline 22 & & & & & 1 & -1 & 1 & 0 & 1 & 0 & 0 & -1 & & & & & & & & \\
\hline 23 & & & & & 1 & -1 & 0 & -1 & 1 & 0 & 0 & 1 & & & & & & & & \\
\hline 24 & & & & & 1 & -1 & 0 & 1 & 1 & 1 & 0 & 0 & & & & & & & & \\
\hline 25 & & & & & 1 & 0 & -1 & -1 & & & & & & & & & & & & \\
\hline 26 & & & & & 1 & 0 & -1 & 1 & & & & & & & & & & & & \\
\hline 27 & & & & & 1 & 0 & 1 & -1 & & & & & & & & & & & & \\
\hline 28 & & & & & 1 & 0 & 1 & 1 & & & & & & & & & & & & \\
\hline 29 & & & & & 1 & 1 & -1 & 0 & & & & & & & & & & & & \\
\hline 30 & & & & & 1 & 1 & 1 & 0 & & & & & & & & & & & & \\
\hline 31 & & & & & 1 & 1 & 0 & -1 & & & & & & & & & & & & \\
\hline 32 & & & & & 1 & 1 & 0 & 1 & & & & & & & & & & & & \\
\hline
\end{tabular}


The first 36-run design, the 68-run design and the two 72-run designs are suited for a cuboidal design region, while the second 36-run design is more suitable for a spherical design region.

\subsection{Equivalent-Estimation Split-Plot Designs Based on Supple- mentary Difference Set Designs}

The second family of equivalent-estimation designs we propose is derived from supplementary difference set designs, the construction of which is outlined in the appendix. The original designs involve two parts:

1. a factorial part, in which each run has one factor acting at its middle level and $M-1$ factors set at either -1 or +1 , and

2. $2 M$ different axial points at a distance $\alpha$ from the center, where the distance $\alpha$ is determined so as to achieve rotatability in completely randomized experiments.

The designs are similar to the subset designs in Gilmour (2006), in the sense that they also include points from the subsets $S_{r}, r=0, \ldots, M$, of the three-level full factorial design. More specifically, they contain points from the subsets $S_{M-1}$ and $S_{1}$. However, the axial distance, which is one in the subset designs, is equal to a certain value $\alpha$ to achieve rotatability in the supplementary difference set designs. Another difference is that the construction method based on supplementary difference sets does not use all the points in $S_{M-1}$, but only a fraction of them. The fact that the designs do not contain points from $S_{M}$ implies that they are not ideal for use with cuboidal design regions. They are better suited for a spherical design region.

The supplementary difference set designs have the same kind of attractive properties as central composite and Box-Behnken designs: (i) all main effects and two-factor interaction effects can be estimated independently, (ii) the designs are factor-wise balanced, (iii) the designs are easy to construct, (iv) they allow the second-order model to be fitted efficiently and result in precise predictions, and, finally, (v) they possess attractive projection properties. The fact that the supplementary difference set designs have other numbers of runs than the central composite and Box-Behnken designs make them attractive in situations where the budgeted number of runs is different from the number of runs in central composite and Box-Behnken designs.

As with the subset designs, in order to construct equivalent-estimation split-plot designs from supplementary difference set designs, we rearrange the runs of the factorial portion and of the axial portion in increasing order of the whole-plot factor levels. The size 
Table 4: Supplementary difference set design with four factors and 40 runs

\begin{tabular}{cccl}
$w_{1}$ & $w_{2}$ & $s_{1}$ & $s_{2}$ \\
\hline $\mathbf{0}_{8}$ & $\pm \mathbf{1}_{8}$ & $\pm \mathbf{1}_{8}$ & $\pm \mathbf{1}_{8}$ \\
$\pm \mathbf{1}_{8}$ & $\mathbf{0}_{8}$ & $\pm \mathbf{1}_{8}$ & $\pm \mathbf{1}_{8}$ \\
$\pm \mathbf{1}_{8}$ & $\pm \mathbf{1}_{8}$ & $\mathbf{0}_{8}$ & $\pm \mathbf{1}_{8}$ \\
$\pm \mathbf{1}_{8}$ & $\pm \mathbf{1}_{8}$ & $\pm \mathbf{1}_{8}$ & $\mathbf{0}_{8}$ \\
$\pm \boldsymbol{\alpha}$ & $\mathbf{0}_{2}$ & $\mathbf{0}_{2}$ & $\mathbf{0}_{2}$ \\
$\mathbf{0}_{2}$ & $\pm \boldsymbol{\alpha}$ & $\mathbf{0}_{2}$ & $\mathbf{0}_{2}$ \\
$\mathbf{0}_{2}$ & $\mathbf{0}_{2}$ & $\pm \boldsymbol{\alpha}$ & $\mathbf{0}_{2}$ \\
$\mathbf{0}_{2}$ & $\mathbf{0}_{2}$ & $\mathbf{0}_{2}$ & $\pm \boldsymbol{\alpha}$ \\
\hline
\end{tabular}

of the whole plots must again be so that the subplot designs are orthogonal. Except for designs with fewer than five factors, the factorial part of supplementary difference set designs is based on a $2^{M-f}$ fractional factorial design of resolution $\mathrm{V}$, in order to guarantee estimability of the second-order model. The axial runs for the subplot factors form a single whole plot. If necessary, the axial points are repeated in order to obtain a balanced design. We did not use the axial points corresponding to whole-plot factors in our construction of equivalent-estimation split-plot designs based on supplementary difference set designs.

Example 3. A design for two whole-plot factors $w_{1}$ and $w_{2}$ and two subplot factors $s_{1}$ and $s_{2}$. We start from the 40-run supplementary difference set design with four factors in Table 4 . In that table, $\mathbf{0}_{8}$ and $\mathbf{0}_{2}$ represent eight- and two-dimensional zero vectors, respectively. Each of the first four rows in the table represents a $2^{4-1}$ fractional factorial design, $\left( \pm \mathbf{1}_{8}, \pm \mathbf{1}_{8}, \pm \mathbf{1}_{8}, \pm \mathbf{1}_{8}\right)$, with one column replaced by zeros. Finally, $\pm \boldsymbol{\alpha}$ is a vector containing the elements $-\alpha$ and $\alpha$.

In order to obtain an equivalent-estimation split-plot design, we rearrange the 32 runs in the factorial part in increasing order of the whole-plot factor levels in the first two columns. This results in eight whole plots of size four. Of the eight axial runs in the original supplementary difference set design, we keep only those four that correspond to the subplot factors. In this way, we eventually obtain the equivalent-estimation split-plot design with nine whole plots of four runs displayed in Table 5. The axial distance $\alpha$ can be set to one without destroying the equivalent-estimation property.

From the family of supplementary difference set designs, we can also construct unbalanced equivalent-estimation split-plot designs. These designs are unbalanced only because the size of the whole plot containing the axial runs is different from the size of the whole plots created from the design's factorial portion. 
Table 5: 36-run equivalent-estimation split-plot design with nine whole plots of size four, two whole-plot factors $w_{1}$ and $w_{2}$, and two subplot factors $s_{1}$ and $s_{2}$.

\begin{tabular}{crrrrrrrrr}
\hline Whole plot & $w_{1}$ & $w_{2}$ & $s_{1}$ & $s_{2}$ & Whole plot & $w_{1}$ & $w_{2}$ & $s_{1}$ & $s_{2}$ \\
\hline 1 & 0 & -1 & -1 & -1 & 6 & 1 & -1 & 0 & 1 \\
1 & 0 & -1 & -1 & 1 & 6 & 1 & -1 & 0 & -1 \\
1 & 0 & -1 & 1 & 1 & 6 & 1 & -1 & -1 & 0 \\
1 & 0 & -1 & 1 & -1 & 6 & 1 & -1 & 1 & 0 \\
\hline 2 & 0 & 1 & -1 & 1 & 7 & -1 & 1 & 0 & 1 \\
2 & 0 & 1 & -1 & -1 & 7 & -1 & 1 & 0 & -1 \\
2 & 0 & 1 & 1 & -1 & 7 & -1 & 1 & -1 & 0 \\
2 & 0 & 1 & 1 & 1 & 7 & -1 & 1 & 1 & 0 \\
\hline 3 & -1 & 0 & -1 & -1 & 8 & 1 & 1 & 0 & -1 \\
3 & -1 & 0 & -1 & 1 & 8 & 1 & 1 & 0 & 1 \\
3 & -1 & 0 & 1 & 1 & 8 & 1 & 1 & -1 & 0 \\
3 & -1 & 0 & 1 & -1 & 8 & 1 & 1 & 1 & 0 \\
\hline 4 & 1 & 0 & -1 & 1 & 9 & 0 & 0 & $-\alpha$ & 0 \\
4 & 1 & 0 & -1 & -1 & 9 & 0 & 0 & $\alpha$ & 0 \\
4 & 1 & 0 & 1 & -1 & 9 & 0 & 0 & 0 & $-\alpha$ \\
4 & 1 & 0 & 1 & 1 & 9 & 0 & 0 & 0 & $\alpha$ \\
\hline 5 & -1 & -1 & 0 & -1 & & & & & \\
5 & -1 & -1 & 0 & 1 & & & & & \\
5 & -1 & -1 & -1 & 0 & & & & & \\
5 & -1 & -1 & 1 & 0 & & & & & \\
\hline & & & & & & & & & \\
\hline
\end{tabular}


Table 6: Supplementary difference set design with five factors and 90 runs.

\begin{tabular}{ccccc}
$w_{1}$ & $w_{2}$ & $s_{1}$ & $s_{2}$ & $s_{3}$ \\
\hline $\mathbf{0}_{16}$ & $\pm \mathbf{1}_{16}$ & $\pm \mathbf{1}_{16}$ & $\pm \mathbf{1}_{16}$ & $\pm \mathbf{1}_{16}$ \\
$\pm \mathbf{1}_{16}$ & $\mathbf{0}_{16}$ & $\pm \mathbf{1}_{16}$ & $\pm \mathbf{1}_{16}$ & $\pm \mathbf{1}_{16}$ \\
$\pm \mathbf{1}_{16}$ & $\pm \mathbf{1}_{16}$ & $\mathbf{0}_{16}$ & $\pm \mathbf{1}_{16}$ & $\pm \mathbf{1}_{16}$ \\
$\pm \mathbf{1}_{16}$ & $\pm \mathbf{1}_{16}$ & $\pm \mathbf{1}_{16}$ & $\mathbf{0}_{16}$ & $\pm \mathbf{1}_{16}$ \\
$\pm \mathbf{1}_{16}$ & $\pm \mathbf{1}_{16}$ & $\pm \mathbf{1}_{16}$ & $\pm \mathbf{1}_{16}$ & $\mathbf{0}_{16}$ \\
$\pm \boldsymbol{\alpha}$ & $\mathbf{0}_{2}$ & $\mathbf{0}_{2}$ & $\mathbf{0}_{2}$ & $\mathbf{0}_{2}$ \\
$\mathbf{0}_{2}$ & $\pm \boldsymbol{\alpha}$ & $\mathbf{0}_{2}$ & $\mathbf{0}_{2}$ & $\mathbf{0}_{2}$ \\
$\mathbf{0}_{2}$ & $\mathbf{0}_{2}$ & $\pm \boldsymbol{\alpha}$ & $\mathbf{0}_{2}$ & $\mathbf{0}_{2}$ \\
$\mathbf{0}_{2}$ & $\mathbf{0}_{2}$ & $\mathbf{0}_{2}$ & $\pm \boldsymbol{\alpha}$ & $\mathbf{0}_{2}$ \\
$\mathbf{0}_{2}$ & $\mathbf{0}_{2}$ & $\mathbf{0}_{2}$ & $\mathbf{0}_{2}$ & $\pm \boldsymbol{\alpha}$ \\
\hline
\end{tabular}

Example 4. A design for two whole-plot factors $w_{1}$ and $w_{2}$ and three subplot factors $s_{1}, s_{2}$ and $s_{3}$. We start from the supplementary difference set design with five five-level factors and 90 runs displayed in Table 6 . In that table, $\mathbf{0}_{16}$ and $\mathbf{0}_{2}$ represent sixteen- and two-dimensional zero vectors, respectively. Each of the first five rows of the table represents a $2^{5-1}$ fractional factorial design of resolution $\mathrm{V},\left( \pm \mathbf{1}_{16}, \pm \mathbf{1}_{16}, \pm \mathbf{1}_{16}, \pm \mathbf{1}_{16}\right.$, $\left.\pm \mathbf{1}_{16}\right)$, with one column replaced by zeros. Again, $\pm \boldsymbol{\alpha}$ is a vector containing the elements $-\alpha$ and $\alpha$.

In order to obtain an equivalent-estimation split-plot design with whole plots of size four from this design, we rearrange the 80 rows in its factorial part in increasing order of the whole-plot factor levels. In this fashion, we initially obtain four groups of twelve runs and four groups of eight runs from the factorial part of the supplementary difference set design. Each of the groups with twelve runs can be split in a group of four runs with $s_{1}=0$, a group of four runs with $s_{2}=0$ and another group of four runs with $s_{3}=0$. The groups of size eight can also be split in groups of four runs. To this end, we use a splitting generator similar to the ones used by Bingham, Schoen and Sitter (2004) for creating minimum aberration split-plot designs and similar to the blocking generators used for regular factorial and fractional factorial designs. The splitting generator is essentially a higher-order interaction contrast column. The splitting generator we use is the column corresponding to the three-factor interaction between the subplot factors. That column, as well as the resulting whole plots of size four, are shown in Table 7. In total, the factorial portion of the supplementary difference set design yields 20 groups of four runs.

Selecting any eight out of the twenty factorial whole plots so that each whole-plot factor level combination is present, and so that the second-order response surface model is es- 
timable, produces an equivalent-estimation split-plot design. The whole plots labeled 1 to 8, when augmented with another whole plot containing the six axial points corresponding to the subplot factors, form such a design. The selection of different whole plots affects the D- and I-efficiency of the design, but not the equivalent-estimation property.

We also obtained split-plot designs with twelve whole plots of four runs and one whole plot of six runs as some combinations of the whole-plot factor levels can be repeated without loosing the equivalent-estimation property.

\section{Results}

In Tables 8 and 9, we provide an overview of the equivalent-estimation designs that we obtained from the subset designs and the supplementary difference set designs, along with the D- and I-efficiencies of the designs relative to D-optimal split-plot designs with the same numbers of runs and whole plots, and the same whole-plot sizes. We would like to stress that the overview in Tables 8 and 9 is by no means exhaustive. Our intention is to show that a broad variety of equivalent-estimation split-plot designs can be constructed from the subset designs and the supplementary difference set designs.

The first and second column of the tables contain the number of runs and number of whole plots of the designs, respectively. The next two columns show the corresponding numbers of whole-plot and subplot factors. The last two columns contain the D- and I-efficiencies of the equivalent-estimation designs relative to the D-optimal designs. For designs that include the vertices of the unit cube or hypercube, the efficiencies were computed assuming a cuboidal design region. For the other designs, we assumed a spherical or hyperspherical design region when computing the D- and I-efficiencies. In Table 8, we indicated in the fifth column what design region was used. Since the efficiencies reported in Table 9 are all for designs obtained from the supplementary difference set designs, all of these were computed assuming a spherical design region.

The relative D-efficiencies we report were computed as

$$
\left\{\frac{\left|\mathbf{X}^{\prime} \mathbf{V}^{-1} \mathbf{X}\right|}{\left|\mathbf{X}_{\mathrm{opt}}^{\prime} \mathbf{V}^{-1} \mathbf{X}_{\mathrm{opt}}\right|}\right\}^{1 / p}
$$

where $\mathbf{X}$ represents the model matrix of the equivalent-estimation design and $\mathbf{X}_{\mathrm{opt}}$ represents the model matrix of the D-optimal split-plot design. The relative I-efficiencies we report were computed as

$$
\frac{\int_{\chi} \mathbf{f}^{\prime}(\mathbf{x})\left(\mathbf{X}_{\mathrm{opt}}^{\prime} \mathbf{V}^{-1} \mathbf{X}_{\mathrm{opt}}\right)^{-1} \mathbf{f}(\mathbf{x}) d \mathbf{x}}{\int_{\chi} \mathbf{f}^{\prime}(\mathbf{x})\left(\mathbf{X}^{\prime} \mathbf{V}^{-1} \mathbf{X}\right)^{-1} \mathbf{f}(\mathbf{x}) d \mathbf{x}}=\frac{\operatorname{tr}\left[\left(\mathbf{X}_{\mathrm{opt}}^{\prime} \mathbf{V}^{-1} \mathbf{X}_{\mathrm{opt}}\right)^{-1} \mathbf{M}\right]}{\operatorname{tr}\left[\left(\mathbf{X}^{\prime} \mathbf{V}^{-1} \mathbf{X}\right)^{-1} \mathbf{M}\right]},
$$


Table 7: Whole plots created from the factorial portion of the supplementary difference set design in Table 6, with an indication of eight whole plots that yield an equivalentestimation split-plot design.

\begin{tabular}{|c|c|c|c|c|c|c|c|c|c|c|c|c|c|}
\hline Whole plot & $w_{1}$ & $w_{2}$ & $s_{1}$ & $s_{2}$ & $s_{3}$ & $s_{1} s_{2} s_{3}$ & Whole plot & $w_{1}$ & $w_{2}$ & $s_{1}$ & $s_{2}$ & $s_{3}$ & $s_{1} s_{2} s_{3}$ \\
\hline & -1 & -1 & -1 & 0 & -1 & 0 & & 0 & 1 & 1 & -1 & 1 & -1 \\
\hline & -1 & -1 & 1 & 0 & 1 & 0 & & 0 & 1 & 1 & 1 & -1 & -1 \\
\hline & -1 & -1 & 1 & 0 & -1 & 0 & & 0 & 1 & -1 & -1 & -1 & -1 \\
\hline & -1 & -1 & -1 & 0 & 1 & 0 & & 0 & 1 & -1 & 1 & 1 & -1 \\
\hline 1 & -1 & -1 & 0 & 1 & -1 & 0 & 5 & 0 & 1 & 1 & -1 & -1 & 1 \\
\hline 1 & -1 & -1 & 0 & 1 & 1 & 0 & 5 & 0 & 1 & -1 & 1 & -1 & 1 \\
\hline 1 & -1 & -1 & 0 & -1 & -1 & 0 & 5 & 0 & 1 & 1 & 1 & 1 & 1 \\
\hline \multirow[t]{9}{*}{1} & -1 & -1 & 0 & -1 & 1 & 0 & 5 & 0 & 1 & -1 & -1 & 1 & 1 \\
\hline & -1 & -1 & -1 & 1 & 0 & 0 & 6 & 1 & -1 & 1 & 0 & -1 & 0 \\
\hline & -1 & -1 & 1 & 1 & 0 & 0 & 6 & 1 & -1 & -1 & 0 & -1 & 0 \\
\hline & -1 & -1 & 1 & -1 & 0 & 0 & 6 & 1 & -1 & -1 & 0 & 1 & 0 \\
\hline & -1 & -1 & -1 & -1 & 0 & 0 & 6 & 1 & -1 & 1 & 0 & 1 & 0 \\
\hline & -1 & 0 & 1 & -1 & 1 & -1 & & 1 & -1 & 0 & 1 & -1 & 0 \\
\hline & -1 & 0 & 1 & 1 & -1 & -1 & & 1 & -1 & 0 & -1 & -1 & 0 \\
\hline & -1 & 0 & -1 & -1 & -1 & -1 & & 1 & -1 & 0 & 1 & 1 & 0 \\
\hline & -1 & 0 & -1 & 1 & 1 & -1 & & 1 & -1 & 0 & -1 & 1 & 0 \\
\hline 2 & -1 & 0 & -1 & 1 & -1 & 1 & & 1 & -1 & 1 & 1 & 0 & 0 \\
\hline 2 & -1 & 0 & 1 & 1 & 1 & 1 & & 1 & -1 & -1 & -1 & 0 & 0 \\
\hline 2 & -1 & 0 & 1 & -1 & -1 & 1 & & 1 & -1 & -1 & 1 & 0 & 0 \\
\hline 2 & -1 & 0 & -1 & -1 & 1 & 1 & & 1 & -1 & 1 & -1 & 0 & 0 \\
\hline 3 & -1 & 1 & 1 & 0 & 1 & 0 & 7 & 1 & 0 & 1 & 1 & -1 & -1 \\
\hline 3 & -1 & 1 & 1 & 0 & -1 & 0 & 7 & 1 & 0 & -1 & -1 & -1 & -1 \\
\hline 3 & -1 & 1 & -1 & 0 & -1 & 0 & 7 & 1 & 0 & -1 & 1 & 1 & -1 \\
\hline \multirow[t]{9}{*}{3} & -1 & 1 & -1 & 0 & 1 & 0 & 7 & 1 & 0 & 1 & -1 & 1 & -1 \\
\hline & -1 & 1 & 0 & -1 & 1 & 0 & & 1 & 0 & 1 & -1 & -1 & 1 \\
\hline & -1 & 1 & 0 & 1 & -1 & 0 & & 1 & 0 & -1 & 1 & -1 & 1 \\
\hline & -1 & 1 & 0 & -1 & -1 & 0 & & 1 & 0 & 1 & 1 & 1 & 1 \\
\hline & -1 & 1 & 0 & 1 & 1 & 0 & & 1 & 0 & -1 & -1 & 1 & 1 \\
\hline & -1 & 1 & 1 & -1 & 0 & 0 & 8 & 1 & 1 & 1 & 0 & -1 & 0 \\
\hline & -1 & 1 & 1 & 1 & 0 & 0 & 8 & 1 & 1 & -1 & 0 & -1 & 0 \\
\hline & -1 & 1 & -1 & -1 & 0 & 0 & 8 & 1 & 1 & 1 & 0 & 1 & 0 \\
\hline & -1 & 1 & -1 & 1 & 0 & 0 & 8 & 1 & 1 & -1 & 0 & 1 & 0 \\
\hline 4 & 0 & -1 & 1 & 1 & -1 & -1 & & 1 & 1 & 0 & -1 & -1 & 0 \\
\hline 4 & 0 & -1 & -1 & -1 & -1 & -1 & & 1 & 1 & 0 & 1 & -1 & 0 \\
\hline 4 & 0 & -1 & -1 & 1 & 1 & -1 & & 1 & 1 & 0 & 1 & 1 & 0 \\
\hline \multirow[t]{5}{*}{4} & 0 & -1 & 1 & -1 & 1 & -1 & & 1 & 1 & 0 & -1 & 1 & 0 \\
\hline & 0 & -1 & -1 & 1 & -1 & 1 & & 1 & 1 & 1 & -1 & 0 & 0 \\
\hline & 0 & -1 & 1 & 1 & 1 & 1 & & 1 & 1 & -1 & 1 & 0 & 0 \\
\hline & 0 & -1 & 1 & -1 & -1 & 1 & & 1 & 1 & 1 & 1 & 0 & 0 \\
\hline & 0 & -1 & -1 & -1 & 1 & 1 & & 1 & 1 & -1 & -1 & 0 & 0 \\
\hline
\end{tabular}


Table 8: Overview, D-efficiencies and I-efficiencies of the equivalent-estimation split-plot designs obtained from subset designs.

\begin{tabular}{ccccccc}
\hline$N$ & $b$ & $M_{w}$ & $M_{s}$ & Region & D-efficiency & I-efficiency \\
\hline $16^{a}$ & 4 & 1 & 2 & spherical & 0.8262 & 1.2454 \\
$20^{a}$ & 5 & 1 & 2 & cuboidal & 0.8482 & 0.3819 \\
$20^{a}$ & 5 & 1 & 2 & cuboidal & 0.7336 & 1.1210 \\
24 & 6 & 1 & 2 & cuboidal & 0.8485 & 1.2905 \\
48 & 6 & 1 & 3 & cuboidal & 0.6895 & 0.3231 \\
48 & 6 & 1 & 3 & spherical & 0.5430 & 1.3858 \\
$36^{b}$ & 9 & 2 & 2 & spherical & 0.9488 & 0.8385 \\
$36^{b}$ & 9 & 2 & 2 & cuboidal & 0.7437 & 1.2708 \\
$72^{b}$ & 9 & 2 & 2 & cuboidal & 0.8992 & 1.5836 \\
$68^{b}$ & 17 & 2 & 2 & cuboidal & 0.8220 & 1.2729 \\
$72^{b}$ & 18 & 2 & 2 & cuboidal & 0.7971 & 1.4359 \\
80 & 10 & 2 & 3 & cuboidal & 0.6878 & 0.8114 \\
80 & 10 & 2 & 3 & spherical & 0.5296 & 1.2842 \\
68 & 17 & 3 & 2 & spherical & 0.7683 & 1.0958 \\
88 & 22 & 3 & 2 & cuboidal & 0.8457 & 0.6825 \\
84 & 21 & 4 & 2 & cuboidal & 0.8910 & 0.6067 \\
84 & 21 & 4 & 2 & cuboidal & 0.7886 & 1.0653 \\
104 & 26 & 4 & 2 & cuboidal & 0.8770 & 0.3945 \\
104 & 26 & 4 & 2 & cuboidal & 0.7827 & 1.2314 \\
\hline$a$ & & & & &
\end{tabular}

${ }^{a}$ See Example 1.

${ }^{b}$ See Example 2. 
Table 9: Overview, D-efficiencies and I-efficiencies of the equivalent-estimation split-plot designs obtained from supplementary difference set designs.

\begin{tabular}{cccccc}
\hline$N$ & $b$ & $M_{w}$ & $M_{s}$ & D-efficiency & I-efficiency \\
\hline 16 & 4 & 1 & 2 & 0.7832 & 1.0083 \\
64 & 4 & 1 & 4 & 0.7212 & 1.1570 \\
$36^{c}$ & 9 & 2 & 2 & 0.9222 & 0.9800 \\
40 & 10 & 2 & 2 & 0.9078 & 1.0565 \\
$38^{d}$ & 9 & 2 & 3 & 0.9405 & 0.9343 \\
44 & 10 & 2 & 3 & 0.7181 & 1.1034 \\
60 & 13 & 2 & 3 & 0.8034 & 1.0450 \\
$54^{d}$ & 13 & 2 & 3 & 0.8005 & 0.9848 \\
144 & 9 & 2 & 4 & 0.8430 & 1.0193 \\
84 & 21 & 3 & 2 & 0.9975 & 0.9533 \\
88 & 22 & 3 & 2 & 1.0015 & 0.9964 \\
166 & 21 & 3 & 3 & 0.9491 & 0.9312 \\
172 & 22 & 3 & 3 & 0.9472 & 0.9643 \\
336 & 21 & 3 & 4 & 0.8944 & 0.9737 \\
352 & 22 & 3 & 4 & 0.8829 & 0.9991 \\
198 & 49 & 4 & 3 & 0.8904 & 0.9119 \\
204 & 50 & 4 & 3 & 1.0583 & 0.8662 \\
\hline
\end{tabular}

${ }^{c}$ See Example 3.

${ }^{d}$ See Example 4. 
where $\chi$ represents the (cuboidal or spherical) design region and $\mathbf{M}$ is the moments matrix,

$$
\mathbf{M}=\int_{\chi} \mathbf{f}(\mathbf{x}) \mathbf{f}^{\prime}(\mathbf{x}) d \mathbf{x}
$$

Expression for calculating the moments matrix are given by Hardin and Sloane (1991a,b).

The balanced D-optimal designs for cuboidal regions we used as benchmarks were obtained using 10000 runs of the coordinate-exchange algorithm of Jones and Goos (2007), as implemented in JMP. The remaining ones were obtained using 10000 runs of the pointexchange algorithm of Goos and Vandebroek (2003).

About half of the designs listed in Table 8 have D-efficiencies above $80 \%$, which we consider satisfactory and which matches the D-efficiencies of equivalent-estimation designs based on central composite and Box-Behnken design reported in Vining, Kowalski and Montgomery (2005) and Parker, Kowalski and Vining (2006, 2007a,b). Remarkably, most of the equivalent-estimation designs we report in Table 8 outperform the D-optimal splitplot designs in terms of I-efficiency. In some case, the designs we constructed are more than $40 \%$ better in terms of I-efficiency than the corresponding D-optimal designs. From the table, it should also be clear that there is an inverse relationship between a design's performance in terms of D-efficiency and its performance in I-efficiency. The designs that have the best relative performance in terms of I-efficiency in general perform very poorly in terms of D-efficiency.

We should also point out that some of the equivalent-estimation designs we constructed perform poorly in terms of both D- and I-efficiency. The design with 48 runs for a cuboidal design region mentioned in Table 8 is an example of a design with a very poor I-efficiency, which is not compensated by a good performance in terms of D-efficiency. The reason for this poor performance is a lack of center points and/or axial points. As a result, these poor performance of these designs can be improved easily by adding center points or axial points.

Given the importance of the performance of response surface designs in terms of prediction variances, it is useful to construct fraction of design space plots to compare alternative design options, and to compare quantiles for the prediction variances. Figure 1 shows fraction of design space plots for three of the cases for which we found good equivalentestimation designs, assuming that $\sigma_{\gamma}^{2}$ and $\sigma_{\epsilon}^{2}$ are both one. Some detailed statistics for the distributions of the prediction variances for the three cases are shown in Table 10.

Each plot in Figure 1 shows the performance of an equivalent-estimation design, represented by the solid line, and the corresponding D-optimal design, represented by the dashed line. The plots provide a more detailed picture of the prediction variances throughout the complete design region than the I-efficiencies reported in Tables 8 and 9. Figure 1a compares the predictive performance of the 24-run equivalent-estimation design involving one hard-to-change factor and two easy-to-change factors in six whole plots of four runs 
Table 10: Minimum and maximum prediction variances, along with several quantiles, for the 24-, 72- and 84-run split-plot designs compared in Figure 1.

\begin{tabular}{lcccccc}
\hline & \multicolumn{2}{c}{24 runs } & \multicolumn{2}{c}{72 runs } & \multicolumn{2}{c}{84 runs } \\
\cline { 2 - 7 } & Eq. Est. & D-opt. & Eq. Est. & D-opt. & Eq. Est. & D-opt. \\
\hline Maximum & 1.5260 & 1.5609 & 0.6798 & 0.7281 & 6.0911 & 1.6372 \\
$97.5 \%$ & 1.1528 & 1.4623 & 0.5046 & 0.6786 & 2.1859 & 1.2524 \\
$75 \%$ & 0.8660 & 1.2908 & 0.3823 & 0.5686 & 0.9808 & 0.9969 \\
Median & 0.7487 & 0.9840 & 0.3409 & 0.5128 & 0.7198 & 0.8925 \\
$25 \%$ & 0.6668 & 0.7535 & 0.3115 & 0.4362 & 0.5480 & 0.7952 \\
$2.5 \%$ & 0.5667 & 0.5362 & 0.2782 & 0.3367 & 0.3417 & 0.6335 \\
Minimum & 0.5338 & 0.4675 & 0.2631 & 0.2758 & 0.2796 & 0.4940 \\
\hline
\end{tabular}

for a cuboidal design region. The equivalent-estimation design clearly has the best overall performance in terms of prediction variance but there is a small fraction of about $10 \%$ of the design space where the D-optimal design gives smaller prediction variances. More specifically, the D-optimal design has a slightly smaller minimum prediction variance and a slightly smaller maximum prediction variance. Figure $1 \mathrm{~b}$ compares the predictive performance of the 72-run equivalent-estimation design involving two hard-to-change factors and two easy-to-change factors in 18 whole plots of four runs. In this case, the fraction of design space plot for the equivalent-estimation design lies completely below that for the D-optimal design, The curve for the equivalent-estimation design is relatively flat, indicating that the prediction variance is quite stable in large parts of the design space. Finally, Figure1b gives the fraction of design space plots for 84 runs and 21 whole plots of size four. In this case, where the I-efficiency of the equivalent-estimation design relative to the D-optimal design is 1.0653 , the added value of the fraction of design space plots is nicely illustrated. The equivalent-estimation design is the better design option in the largest part of the design space. In more than $75 \%$ of the design space, the equivalent-estimation design gives the better prediction variances. Also, $50 \%$ of the smallest prediction variances for the equivalent-estimation design are $20 \%$ or more smaller than those for the D-optimal design. However, this good performance comes at the expense of large prediction variances in other parts of the design space. Therefore, in this case, we would recommend using the equivalent-estimation design only if the equivalent-estimation property is of crucial importance to the practitioner, and if the optimum settings of the process under investigation are expected to be in the part of the design space that has low prediction variance (which is about $75 \%$ of the design space).

Table 9 shows that the equivalent-estimation designs we constructed based on supplementary difference set designs in general have better relative D-efficiencies than those 


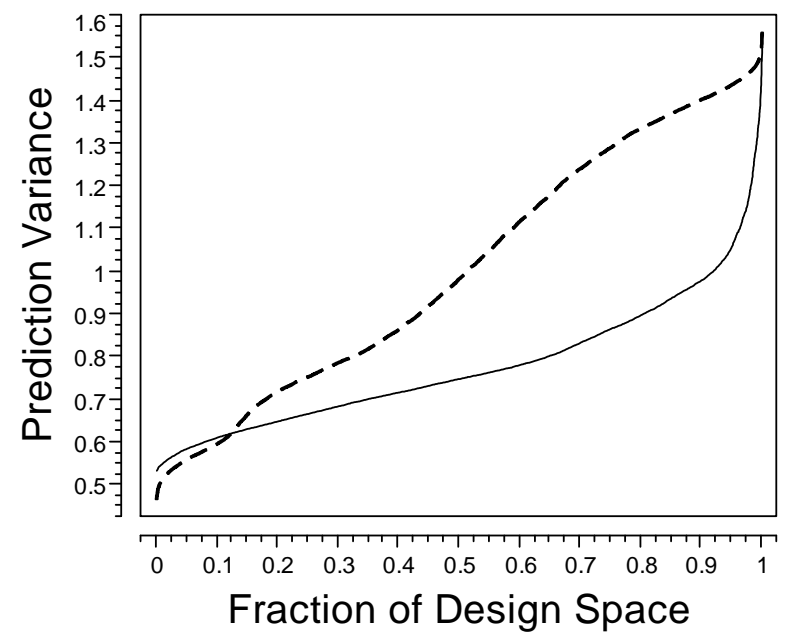

(a) 24 runs: Six whole plots of four runs

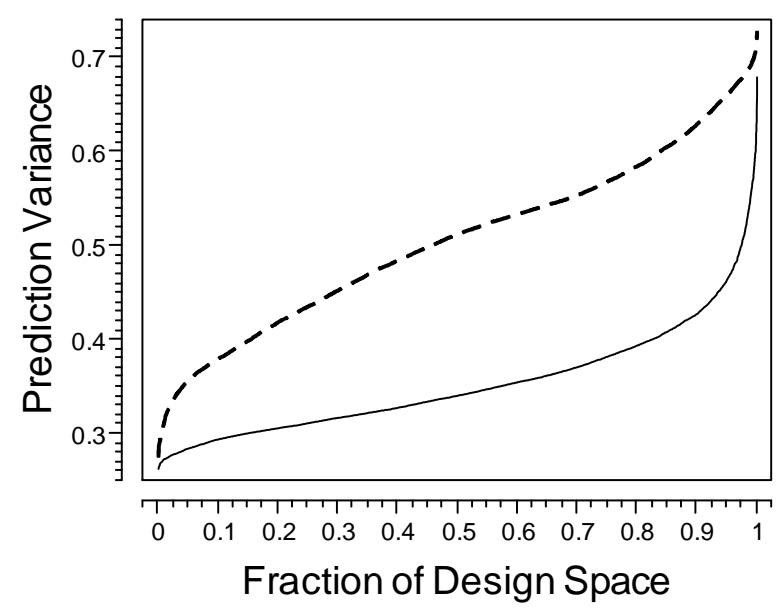

(b) 72 runs: 18 whole plots of four runs

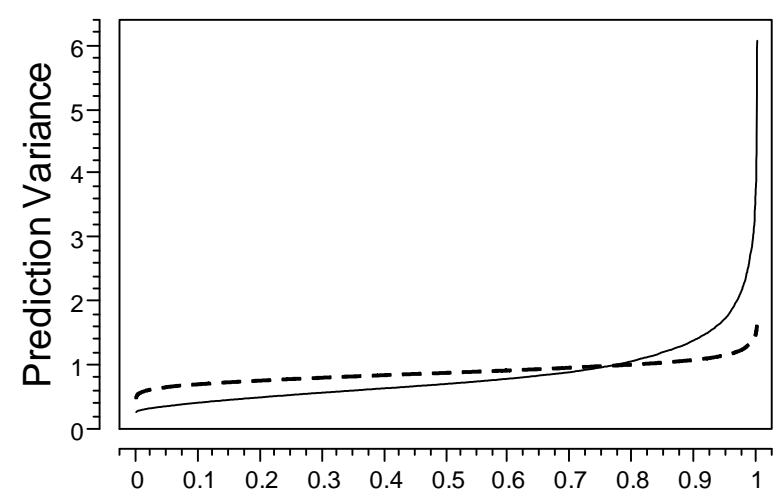

Fraction of the Design Space

(c) 84 runs: 21 whole plots of four runs

Figure 1: Fraction of design space plots comparing equivalent-estimation designs from Table 8 (solid lines) and D-optimal designs (dashed lines). 
based on subset designs, as well as high relative I-efficiencies. Here too, we can observe an inverse relationship between a design's performance in terms of D-efficiency and its performance in terms of I-efficiency.

Note that, in two cases, for 88 runs with $M_{w}=3$ and $M_{s}=2$ and for 204 runs with $M_{w}=4$ and $M_{s}=3$, the equivalent-estimation designs we constructed based on supplementary difference set designs outperform the designs produced by the point-exchange algorithm in terms of D-efficiency, even after 100000 starts of the algorithm.

\section{Summary}

Split-plot designs are very effective in reducing the cost of an experiment in the presence of hard-to-change factors. However, in general, the randomization structure of a splitplot design requires the use of generalized least squares to estimate the model. This estimation approach is not implemented in some software packages, which has led to the development of various methods for constructing split-plot designs for which the ordinary and generalized least squares estimators produce the same point estimates.

In this paper, we have shown how to modify two families of second-order response surface designs in order to obtain equivalent-estimation split-plot designs. We have provided a broad range of new equivalent-estimation designs, with different numbers of runs and whole plots than the equivalent-estimation designs reported in Vining, Kowalski and Montgomery (2005) and Parker, Kowalski and Vining (2006, 2007a,b). Hence, we offer practitioners without access to advanced software an additional set of split-plot designs to choose from. An additional advantage is that most of the designs are highly D- and/or

I-efficient. Some of the designs we obtained outperformed D-optimal designs by more than $40 \%$ in terms of I-efficiency.

The two families of the used response surface designs are very broad, and the proposed construction method can be easily applied for equivalent-estimation designs with sizes of whole plots and numbers of whole-plot and subplot factors other than those reported here. The designs discussed here are only a small representative sample of all possible equivalent-estimation designs one can obtain, because both the subset designs and the supplementary difference set designs are essentially infinite classes of response surface designs, which possess the same kinds of attractive properties as central composite and Box-Behnken designs. It is possible to increase the axial distances in the designs that we propose so as to increase the D-efficiencies.

Some of the equivalent-estimation designs we report involve large numbers of runs. We do not consider these large numbers of runs an important drawback because, in split-plot experiments, it is mainly the number of whole plots that drives the cost of the experiment 
and not the number of runs.

An Excel sheet with all the designs reported in this paper is available from the authors.

\section{Appendix: Construction of supplementary difference set}

\section{designs}

The construction method of the supplementary difference sets, along with the theoretical underpinnings for it, are described in detail in a hitherto unpublished paper by Koukouvinos, Mylona and Skountzou. While the justification for the construction method is highly mathematical, the construction of the supplementary difference set designs is relatively simple. In this appendix, we provide a much abbreviated example-based description of the construction. The supplementary difference set designs possess five levels for each factor and were developed to be used for experiments with complete randomization. Under complete randomization, the designs are rotatable.

The supplementary difference set designs are composed of two parts, a factorial part, in which all factors take the levels $-1,0$ and +1 , and an axial part involving axial runs similar to those in a central composite design. A key requirement for the supplementary difference set designs for five or more factors is that their factorial part is based on fractional factorial designs of resolution $\mathrm{V}$ in order to attain the rotatability of the design. For designs with three or four factors, a fraction with a smaller resolution can be used. The axial distance should equal

$$
\alpha=\sqrt[4]{(2 k-5) \times 2^{k-f-1}}
$$

where $k$ is the number of factors and $f$ indicates what fraction of a full factorial design is used in the construction. In general, the supplementary difference set designs involve $k \times 2^{k-f}+2 k$ runs.

Suppose we want to construct a small supplementary difference set design with $k=3$ factors. For the factorial part of the design, we can start from the 4-run $2^{3-1}$ fractional factorial design. To obtain a three-factor supplementary difference set design, we have to arrange three copies of the fractional factorial design underneath each other. The resulting design is shown in the left panel of Table 11. The next step is to replace the $i$ th column of the $i$ th copy of the fractional factorial design by a column of zeros. This leads to the modified design in the right panel of Table 11. A short-hand notation for the modified design is

$$
\left[\begin{array}{ccc}
\mathbf{0}_{4} & \pm \mathbf{1}_{4} & \pm \mathbf{1}_{4} \\
\pm \mathbf{1}_{4} & \mathbf{0}_{4} & \pm \mathbf{1}_{4} \\
\pm \mathbf{1}_{4} & \pm \mathbf{1}_{4} & \mathbf{0}_{4}
\end{array}\right]
$$


Table 11: Construction of the factorial part of a three-factor supplementary difference set design. The left panel shows three replicates of the $2^{3-1}$ fractional factorial design. The right panel shows how the initial two-level design is modified into the three-level factorial part of the supplementary difference set design.

\begin{tabular}{rrr|rrr}
\hline \multicolumn{3}{c|}{ Initial } & \multicolumn{3}{c}{ Modified } \\
\hline$x_{1}$ & $x_{2}$ & $x_{3}$ & $x_{1}$ & $x_{2}$ & $x_{3}$ \\
\hline-1 & -1 & 1 & 0 & -1 & 1 \\
1 & -1 & -1 & 0 & -1 & -1 \\
-1 & 1 & -1 & 0 & 1 & -1 \\
1 & 1 & 1 & 0 & 1 & 1 \\
\hline-1 & -1 & 1 & -1 & 0 & 1 \\
1 & -1 & -1 & 1 & 0 & -1 \\
-1 & 1 & -1 & -1 & 0 & -1 \\
1 & 1 & 1 & 1 & 0 & 1 \\
\hline-1 & -1 & 1 & -1 & -1 & 0 \\
1 & -1 & -1 & 1 & -1 & 0 \\
-1 & 1 & -1 & -1 & 1 & 0 \\
1 & 1 & 1 & 1 & 1 & 0 \\
\hline & & &
\end{tabular}

where $\mathbf{0}_{4}$ represents a four-dimensional zero vector. Each of the rows in the table represents a $2^{3-1}$ fractional factorial design, $\left( \pm \mathbf{1}_{4}, \pm \mathbf{1}_{4}, \pm \mathbf{1}_{4}\right)$, with one column replaced by zeros.

The final step in the construction is to add the axial points. Since $k=3$ and $f=1$, the required axial distance is

$$
\alpha=\sqrt[4]{(2 \times 3-5) \times 2^{3-1-1}}=\sqrt[4]{2}=1.1892 .
$$

The resulting design has $3 \times 2^{3-1}+3 \times 2=18$ runs in total. The complete design is given 
by

$$
\left[\begin{array}{ccc}
\mathbf{0}_{4} & \pm \mathbf{1}_{4} & \pm \mathbf{1}_{4} \\
\pm \mathbf{1}_{4} & \mathbf{0}_{4} & \pm \mathbf{1}_{4} \\
\pm \mathbf{1}_{4} & \pm \mathbf{1}_{4} & \mathbf{0}_{4} \\
\pm \boldsymbol{\alpha} & \mathbf{0}_{2} & \mathbf{0}_{2} \\
\mathbf{0}_{2} & \pm \boldsymbol{\alpha} & \mathbf{0}_{2} \\
\mathbf{0}_{2} & \mathbf{0}_{2} & \pm \boldsymbol{\alpha}
\end{array}\right]
$$

where $\mathbf{0}_{2}$ represents a two-dimensional zero vector and $\pm \boldsymbol{\alpha}$ is a vector containing the elements $-\alpha$ and $\alpha$.

The factorial part of the supplementary difference set designs resembles a Box-Behnken design, which is also characterized by the fact the factors are not all at either their low level or their high level. A difference between the factorial part of supplementary difference set designs and Box-Behken designs is that, in the former, exactly one of the factors is at its middle level, while, in the latter, the number of factors at their middle level increases with the number of factors. A feature that the supplementary difference set designs have in common with central composite designs is that they involve axial points. Unlike central composite and Box-Behnken designs, supplementary difference set designs do not involve center points.

An attractive property of completely randomized supplementary difference set designs is that they perform well in terms of the I-optimality criterion. In other words, they result in small average prediction variances. As we have shown in the main text, the equivalentestimation split-plot designs we constructed using supplementary difference set designs also perform well in terms of this criterion. Unlike their completely randomized counterparts, the split-plot supplementary difference set designs are not rotatable. However, achieving rotatability for split-plot designs is practically infeasible, as very large axial distances are required for that purpose. This was shown by Wang, Vining and Kowalski (2010). We do not consider the lack of rotatability a problem: in this paper, we are primarily interested in achieving designs that possess the equivalent-estimation property. A secondary goal is achieving low variances for the factor-effect estimates and prediction variances. Whether or not the prediction variance is constant on concentric spheres around the design region's center is at best a tertiary issue.

\section{References}

Ahmad, T. and Gilmour, S. G. (2010). Robustness of subset response surface designs to missing observations, Journal of Statistical Planning and Inference 140: 92-103.

Bingham, D. R., Schoen, E. D. and Sitter, R. R. (2004). Designing fractional factorial splitplot experiments with few whole-plot factors, Journal of the Royal Statistical Society, Ser. C (Applied Statistics) 53: 325-339. Corrigendum, 54, 955-958. 
Gilmour, S. G. (2006). Response surface designs for experiments in bioprocessing, Biometrics 62: 323-331.

Goos, P. (2002). The Optimal Design of Blocked and Split-plot Experiments, New York: Springer.

Goos, P. (2006). Optimal versus orthogonal and equivalent-estimation design of blocked and split-plot experiments, Statistica Neerlandica 60: 361-378.

Goos, P. and Vandebroek, M. (2001). Optimal split-plot designs, Journal of Quality Technology 33: 436-450.

Goos, P. and Vandebroek, M. (2003). D-optimal split-plot designs with given numbers and sizes of whole plots, Technometrics 45: 235-245.

Hardin, R. H. and Sloane, N. J. A. (1991a). Computer-generated minimal (and larger) response-surface designs: (II) The cube. http://www2.research.att.com/ njas/doc/meatball.pdf.

Hardin, R. H. and Sloane, N. J. A. (1991b). Computer-generated minimal (and larger) response-surface designs: (II) The sphere. http://www2.research.att.com/ njas/doc/doeh.pdf.

Jones, B. and Goos, P. (2007). A candidate-set-free algorithm for generating D-optimal split-plot designs, Journal of the Royal Statistical Society, Ser. C (Applied Statistics) 56: $347-364$.

Jones, B. and Nachtsheim, C. J. (2009). Split-plot designs: What, why, and how, Journal of Quality Technology 41: 340-361.

Khuri, A. I. and Cornell, J. A. (1996). Response Surfaces, 2nd edn, New York: Dekker.

Letsinger, J. D., Myers, R. H. and Lentner, M. (1996). Response surface methods for bi-randomization structures, Journal of Quality Technology 28: 381-397.

Macharia, H. and Goos, P. (2010). D-optimal and D-efficient equivalent-estimation secondorder split-plot designs, Journal of Quality Technology 42: 358-372.

McElroy, F. W. (1967). A necessary and sufficient condition that ordinary least squares estimators be best linear unbiased, Journal of the American Statistical Association 62: 1302-1304.

Myers, R. H. and Montgomery, D. C. (2002). Response Surface Methodology: Process and Product Optimization Using Designed Experiments, 2nd edn, New York: Wiley.

Parker, P. A., Kowalski, S. M. and Vining, G. G. (2006). Classes of split-plot response surface designs for equivalent estimation, Quality and Reliability Engineering International 22: 291-305. 
Parker, P. A., Kowalski, S. M. and Vining, G. G. (2007a). Construction of balanced equivalent estimation second-order split-plot designs, Technometrics 49: 56-65.

Parker, P. A., Kowalski, S. M. and Vining, G. G. (2007b). Unbalanced and minimal point equivalent estimation second-order split-plot designs, Journal of Quality Technology 39: $376-388$.

Vining, G. G., Kowalski, S. M. and Montgomery, D. C. (2005). Response surface designs within a split-plot structure, Journal of Quality Technology 37: 115-129.

Wang, L., Vining, G. G. and Kowalski, S. M. (2010). Two-strata rotatability in splitplot central composite designs, Applied Stochastic Models in Business and Industry 26: 431-447. 